\title{
Global warming: Evidence from satellite observations
}

\author{
C. Prabhakara I, R. Iacovazzi, Jr. ${ }^{2}$, J.-M. Yoo ${ }^{3}$, and G. Dalu 4
}

Abstract. Observations made in Channel $2(53.74 \mathrm{GHz})$ of the Microwave Sounding Unit (MSU) radiometer, flown onboard sequential, sun-synchronous, polar-orbiting NOAA operational satellites, indicate that the mean temperature of the atmosphere over the globe increased during the period 1980 to 1999. In this study, we have minimized systematic errors in the time series introduced by satellite orbital drift in an objective manner. This is done with the help of the onboard warm-blackbody temperature, which is used in the calibration of the MSU radiometer. The corrected MSU Channel 2 observations of the NOAA satellite series reveal that the vertically-weighted global-mean temperature of the atmosphere, with a peak weight near the mid troposphere, warmed at the rate of $0.13 \pm 0.05 \mathrm{Kdecade}^{-1}$ during 1980 to 1999. The global warming deduced from conventional meteorological data that have been corrected for urbanization effects agrees reasonably with this satellite-deduced result.

\section{Introduction}

Meteorological measurements of air temperature over land, taken about a meter above the ground, and ocean surface temperature are commonly used in the estimation of global temperature and its long-term trend (see for e.g. Hansen et al., 1996 and Jones et al., 1999). However, since these conventional data represent point values (i.e., not spatial averages) they may overemphasize adverse effects due to urbanization (Hansen et al., 1999), land-use, and deforestation.

According to Gaffen et al. (2000), conventional meteorological data from radiosondes are "primarily operational observations, and it is not clear that they are of sufficient quality for precise monitoring of climate change." The analysis of Hurrell et al. (2000) also reinforces this viewpoint. For these reasons, we are not comparing the global temperature trend derived here with that of radiosondes.

Observations made by the Microwave Sounding Unit (MSU) radiometer onboard NOAA operational polar-orbiting satellites represent averages over radiometer footprints, each of which has an area of about $10^{4} \mathrm{~km}^{2}$. Also, MSU data coverage is uniform over land and ocean. For these reasons, satellite data are potentially valuable in monitoring global temperature.

Satellite radiometer measurements of surface temperature in the infrared or microwave spectral regions are significantly contaminated by either clouds or surface emissivity. In order to minimize such contamination, Spencer and Christy (1990, here after SC) used the measurements made by the MSU radiometer in Channel 2 ( $\mathrm{Ch}$ 2) centered over a narrow spectral interval near $53.74 \mathrm{GHz}$ of the oxygen absorption band. Each Ch 2 observation reflects the vertically-weighted mean temperature of the atmosphere, with a peak weight near the mid

${ }^{1}$ NASA/Goddard Space Flight Center, Greenbelt, MD

${ }^{2}$ Raytheon ITSS, Lanham, MD

${ }^{3}$ EWHA Womans University, Seoul, South Korea

${ }^{4} \mathrm{CNR}$, Cagliari, Italy troposphere, and is highly correlated with the surface temperature. The study of SC revealed that the MSU Ch 2 data have the potential to monitor global temperature. However. problems in the MSU Ch 2 global temperature time series, arising from merging measurements of independent satellites, have been critically discussed in the studies of Trenberth and Hurrell (1997) and Hurrell and Trenberth (1997, 1998). Also, recent studies (Christy et al., 1998 and Prabhakara and lacovazzi, 1999) have indicated that there are some systematic errors in the MSU data, and these errors have to be removed to infer the global temperature trend.

In this study, the time series of MSU Ch 2 data during the period 1980 to 1999 is deduced from the sequential satellites NOAA $6,7,9,10,11,12$, and 14 . The morning satellites in this sequence, NOAA 6,10 , and 12 , have orbits with Local Equatorial Crossing Time (LECT) around $7 \mathrm{am} / 7 \mathrm{pm}$. The afternoon satellites, NOAA $7,9,11$, and 14 , have orbits with LECT around $2 \mathrm{am} / 2 \mathrm{pm}$.

In order to avoid spurious effects due to satellite altitude change with time, from the 11 measurements of each MSU cross-track scan we use only the nadir measurement (Prabhakara et al., 1998 and Wentz and Schabel, 1998). From all the nadir data of each satellite, we generate monthly-mean Ch 2 temperature, $T$, averaged over the globe with cosine weighting according to latitude. In our analysis, when we need to minimize the annual cycle in $\mathrm{T}$, we introduce the 12 -month running mean temperature, $T^{*}$.
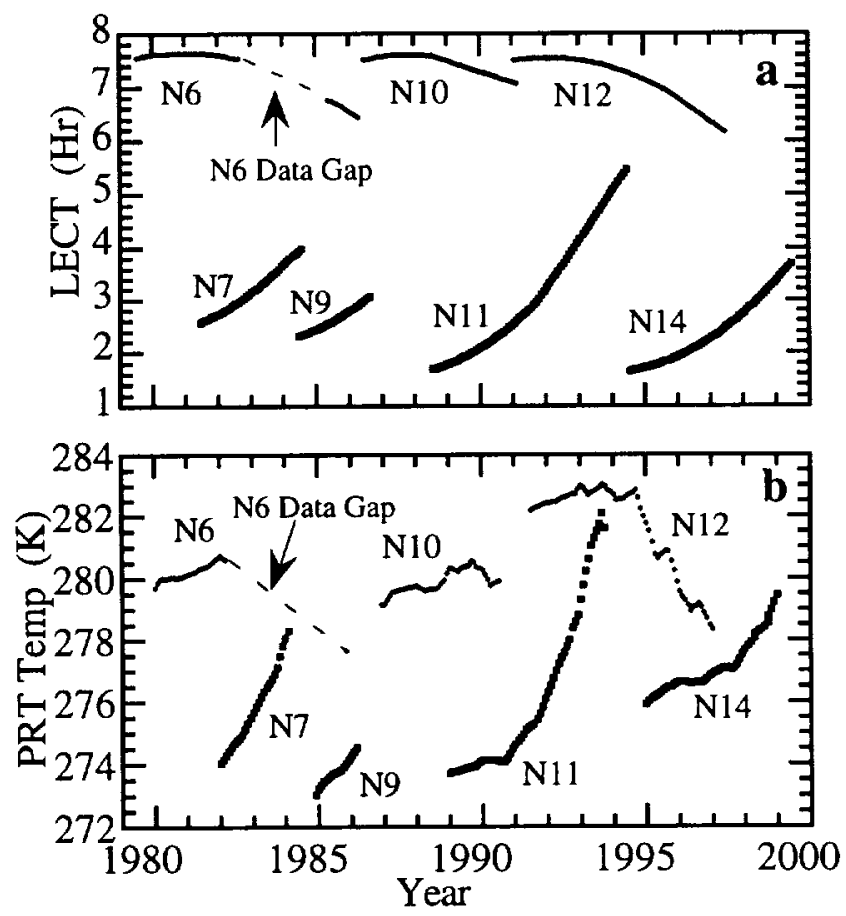

Figure 1: a) Temporal change in LECT of each satellite, b) Temporal change in $T^{*}$ wB of each satellite. The NOAA 6 data gap is indicated in the figures. 


\section{MSU data calibration considerations}

The MSU radiometer flown on operational satellites NOAA 6 through NOAA 14 is of the Dicke type. This radiometer is designed to have calibration capability onboard the satellite with the help of two references - a warm blackbody imbedded in the instrument and free space. The temperature of the warm blackbody $\left(\mathbf{T}_{\mathbf{W B}}\right)$ is monitored by Platinum Resistance Thermometers (PRTs), while free space is assumed to have a temperature of $2.7 \mathrm{~K}$.

Conforming with observations made in a laboratory thermal vacuum chamber, NOAA has developed an empirical MSU calibration equation that is weakly non-linear to estimate the radiance received from an object. The quantities that enter this equation are the warm-blackbody and free-space radiances, as well as the MSU radiometer measured digital counts of the a) cold blackbody, b) warm blackbody, and c) object. For a brief account of the MSU instrument and its calibration procedure, see Mo (1995). In our study, we have applied this procedure (see also NOAA PODUG, 1998) to MSU observations made from NOAA satellites to derive the radiance received from each earth scene. For our purpose, this radiance is converted to brightness temperature.

The analysis made by Mo (1995) shows that this empirical calibration procedure leads to small systematic errors in the data that can be attributed to perturbations in the temperature of the warm or cold blackbodies. In Fig. la, the monthlyaveraged LECT of each NOAA satellite is shown to drift with time, which is due to systematic changes in orbital geometry. From this figure, we may note that the morning satellites drift slowly by about an hour toward the west (earlier local time), while the afternoon satellites drift more rapidly toward the east (later local time). Apparently, for each satellite this change in LECT causes the solar illumination on the satellite to vary, and thereby perturbs the thermal state of the satellite. These thermal perturbations are revealed by the PRT data of the onboard warm blackbody.

\section{Diagnosis of time-dependent errors in MSU data}

While a satellite is in orbit around the earth, on average the temperature of its warm blackbody, $\mathbf{T}_{\mathbf{W B}}$, changes gradually by a small amount $(<10 \mathrm{~K})$ due to drift in LECT over a period of several years. This can be inferred by examination of Fig. Ia, and the 12-month running mean of warm-blackbody temperature, $\mathbf{T}^{*}$ WB , shown in Fig. $1 \mathrm{~b}$. Since warm-blackbody temperature is one of the parameters in the non-linear calibration equation, such perturbations may lead to a systematic time-dependent calibration error, $\varepsilon_{\mathfrak{c}}$, in $\mathbf{T}$.

Also, due to gradual drift in the LECT of a satellite, there is another time-dependent error, $\varepsilon_{\mathbf{d}}$, in $\mathbf{T}$. This error arises because of the earth's diurnal temperature cycle. Thus, there is imbedded in the MSU temperature data a combined error, $\varepsilon$, that is the sum of $\varepsilon_{c}$ and $\varepsilon_{d}$. In this study, we estimate empirically the combined error $\varepsilon$ using MSU observations made from co-orbiting satellites. We may note that Christy et al. (2000) attempt to determine the errors $\varepsilon_{\mathfrak{c}}$ and $\varepsilon_{d}$ separately. They estimate the diurnal-cycle error $\varepsilon_{\mathrm{d}}$ using the difference between the data of the endpoints of the MSU crosstrack scan. Also, they assume that averaging this difference for the ascending and descending legs of the satellite orbits

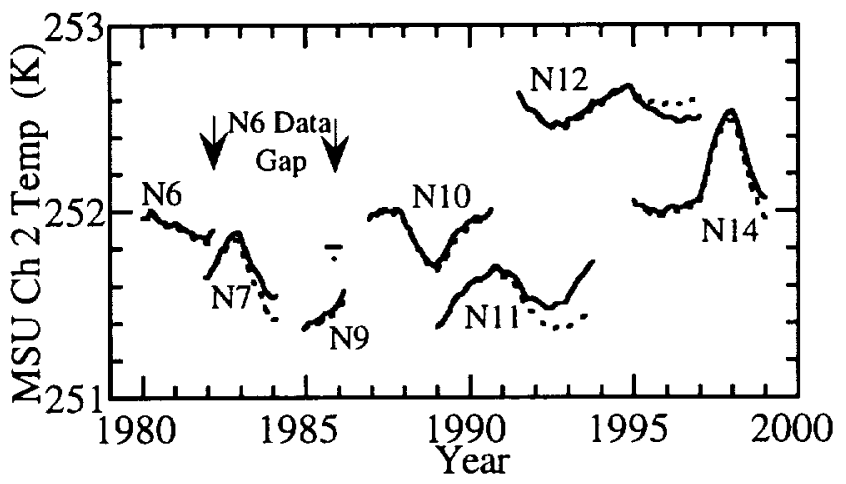

Figure 2: $T^{*}$ versus Year for NOAA 6 through NOAA 14. The solid lines represent uncorrected $T^{*}$, while the dashed lines represent $T^{*}$ corrected for drift effects.

will eliminate cross-swath biases. We find that such averaging does not eliminate cross-swath biases. So, we don't apply Christy et al.'s method to correct the MSU data for the driftrelated errors.

Besides the error $\varepsilon$ in the MSU data of each satellite, there are offsets, denoted by $\delta T^{*}$, between the data of successive satellites. In Fig. 2, we show the 12-month running mean temperature, $T^{*}$, of each satellite to reveal qualitatively the inter-satellite data offsets $\delta T^{*}$. As a prerequisite to estimate these inter-satellite data offsets, first we have to remove $\varepsilon$ from the data of each satellite. Only then we can remove $\delta T^{*}$ and splice the multi-satellite MSU Ch 2 data to get a time series. The global temperature trend can be estimated from such a time series. Splicing of successive satellite data is done using one year of overlap data, except in bridging NOAA 9 and 10 . This particular overlap consists of only three months of data. Since the overlap between NOAA 7 and 9 is very short ( $\sim 45$ days), these overlap data are not used to bridge the data of NOAA 7 and 9. Instead, NOAA 6 data is used. Note that the NOAA 6 data flow was interrupted in April of 1983, but was restored in October of 1985 to provide about a year of overlap data between NOAA 6 and 9 (see Fig. la).

In order to emphasize the effect of $\varepsilon$ on the time series and the trend, we generate two widely different 20 -year MSU Ch 2 times series, $\mathbf{A}$ and $\mathbf{B}$. These two series contain different number of years of uncorrected morning and afternoon satellite data. Time Series $\mathbf{A}$ has the longest possible data record from afternoon satellites, 15 years; and 5 years of data from morning satellites. Time Series $B$ has the shortest possible data record from afternoon satellites, 7 years; and 13 years of data from morning satellites. In Fig. 3, we show anomalies of $\mathbf{T}$ deduced from Time Series $\mathbf{A}$ and $\mathbf{B}$. The global temperature trend derived from $\mathbf{A}$ is $0.24 \mathrm{Kdecade}^{-1}$. On the other hand, the trend from $\mathbf{B}$ is $0.12 \mathrm{Kdecade}^{-1}$. Ideally, the trend estimate should not depend on the length of morning or afternoon satellite data This demonstrates that because of $\varepsilon$, the global temperature trends for Time Series $\mathbf{A}$ and $\mathbf{B}$ differ significantly.

\section{Removal of time-dependent errors and estimation of global temperature trend}

In Fig. $1 \mathrm{~b}, \mathbf{T}^{*}$ WB $_{\text {B }}$ of each satellite is shown as a function of time. For the morning satellites, NOAA 6,10 , and 12 , 


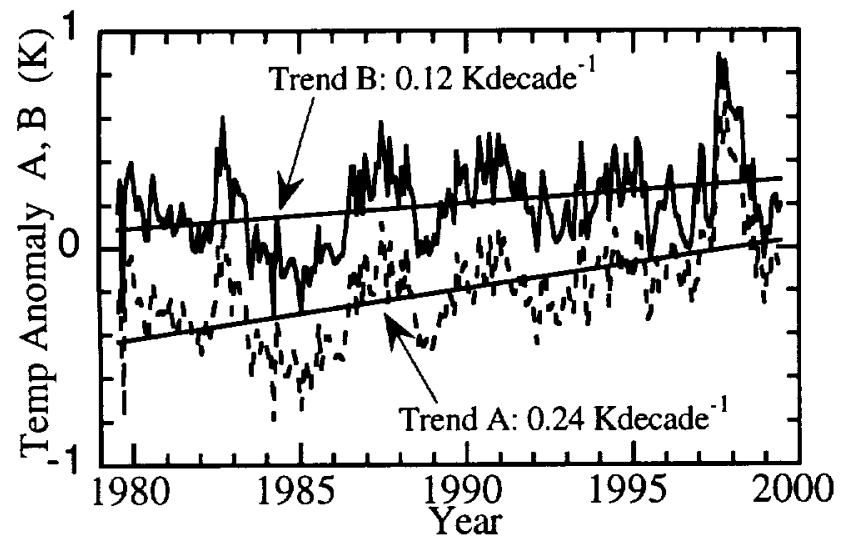

Figure 3: Uncorrected MSU Ch 2 Temperature Anomaly Time Series $\mathbf{A}$ and $\mathbf{B}$ and their trends. For clarity, Time Series $A$ and $B$ are offset by $0.4 \mathrm{~K}$.

$T^{*}$ WB varies systematically with a tendency to decrease steeply after the first few years. This is clearly seen from the relatively long time records of NOAA 6 and 12 . However, $T^{*}$ wB of the afternoon satellites, NOAA $7,9,11$, and 14 , tends to increase systematically with time. We may point out that the opposing trends in $\mathbf{T}^{*}{ }_{\mathrm{WB}}$ of the morning and afternoon satellites are analogous to the time trend of the drift in the LECT shown in Fig. 1a. We find the correlation between 12-month running mean LECT and $T^{*}$ WB for the morning satellites is on average about 0.9 . For the afternoon satellites, this correlation is about 0.96 . These correlations indicate a definite connection between LECT and $\mathbf{T}^{*}$ WB .

In addition to the link between $\mathbf{T}^{*} \mathrm{WB}_{\mathrm{B}}$ and LECT, we also find that the difference $\delta T^{*}{ }_{W B}$ between morning and afternoon satellite data during the time period when they overlap with one another has useful information. Specifically, $\delta T^{*}{ }_{\text {WB }}$ is highly correlated with the corresponding 12-month running mean brightness temperature difference, $\delta T^{*}$, of two satellites during their overlap period. In Fig. 4, we show a regression relationship between $\delta T^{*}{ }_{W B}$ and $\delta T^{*}$ taken from a total of nine years of overlap data of a) NOAA 10 and $11, b$ ) NOAA 11 and 12 , and c) NOAA 12 and 14 . Each of these overlap datasets has a systematic shift with respect to the other. In Fig. 4, such systematic shifts have been eliminated. We find the correlation coefficient between the two variables, $\delta T^{*}{ }_{W_{B}}$ and $\delta T^{*}$, taken from the three overlaps is 0.98 and the slope of the regression line is 0.031 . We can express this regression relationship as:

$$
\delta T^{*}=C_{0}+S \delta T^{*} W_{B}
$$

where the intercept is $C_{0}$ and the slope $S$ is equal to 0.031 . In this analysis, $\delta T^{*}$ versus $\delta T^{*}$ wB of the remaining three overlaps - NOAA 6 to 7, NOAA 6 to 9 , and NOAA 9 to 10 - is not done, as those overlaps of $\mathbf{T}^{*}{ }_{\mathrm{WB}}$ or $\mathrm{T}^{*}$ are of shorter duration.

If there are no drift-related errors in the $\mathrm{Ch} 212$-month running-mean temperature measured by both the satellites during the overlap period, we expect from Eq. 1 that the difference $\delta T^{*}$ between the two satellites should be equal to some constant during that period. However, we know from the Ch 2 records of NOAA $10,11,12$, and 14 that this is not true, and hence we infer there are drift-related errors in these satellite data. These drift-related errors are reflected by the term S $\delta T^{*}{ }_{w B}$ in Eq. 1. From a separate analysis of the data of the three individual overlaps mentioned above, we find the slope $S$ of the regression line between $\delta \mathrm{T}^{*}$ and $\delta \mathrm{T}^{*}$ WB has a mean value of about 0.03 , with a variability of about $10 \%$. We have adopted this mean value of 0.03 for $S$ to estimate the driftrelated errors of all the satellites.

Based on the reasoning presented above and the analysis leading to $\mathrm{Eq}$. 1, we can quantify the drift-related error $\varepsilon(t)$ in Ch 2 data of a satellite with the help of drift-related change in $\mathbf{T}^{*}$ wB. For example, this error in the Ch 2 data of NOAA 11 , $\varepsilon_{11}(t)$, can be estimated from the following equation:

$$
\varepsilon_{11}(t)=S\left[T^{*}{ }_{11 W B}(t)-T^{*}{ }_{11 W B}\left(t_{o}\right)\right] .
$$

In Eq. 2, $t_{0}$ refers to the starting time of the NOAA 11 satellite, and $t$ is some later time. Note, $\varepsilon_{11}\left(t_{0}\right)$ is equal to zero. As seen in Fig. $1 \mathrm{~b}, \mathrm{~T}^{*}{ }_{\mathrm{WB}}$ changes by about $8 \mathrm{~K}$ in five years for NOAA 11 . If not corrected, this leads to an $\varepsilon_{11}(t)$ of about $0.24 \mathrm{~K}$ by the end of the five-year data record. However, we infer the cumulative error of all the satellites is significantly smaller than $0.24 \mathrm{~K}$, because of opposing trends in $T^{*}$ WB $_{B}$ of the morning and afternoon satellites (see Fig. Ib). This inference is consistent with the analysis of Time Series $\mathbf{A}$ and $\mathbf{B}$ presented in the previous section.

Using the mean value of $S$ equal to 0.03 , the monthly-mean temperatures $\mathbf{T}$ of each satellite are corrected for drift-related errors following the example of Eq. 2. The 12-month running mean of corrected $\mathbf{T}$ for each satellite is shown in Fig. 2 with dashed lines. After correction, the intersatellite data offsets are removed. When Time Series $\mathbf{A}$ and $\mathbf{B}$ (see Section 3) are constructed in the above manner, the trends deduced from both times series are close to $0.13 \mathrm{Kdecade}^{-1}$ and they differ by about $0.01 \mathrm{Kdecade}^{-1}$. This demonstrates the sensitivity of the trend to the record lengths of corrected data used to make these two widely different time series.

We have also examined the sensitivity of the trend to the value of S. For Time Series A, we find when $S$ is 0.027 that the trend is close to $0.141 \mathrm{Kdecade}^{-1}$, while when $S$ is 0.033 the trend is close to $0.118 \mathrm{Kdecade}^{-1}$. Slightly less sensitivity to the value of $S$ is found for Time Series $\mathbf{B}$.

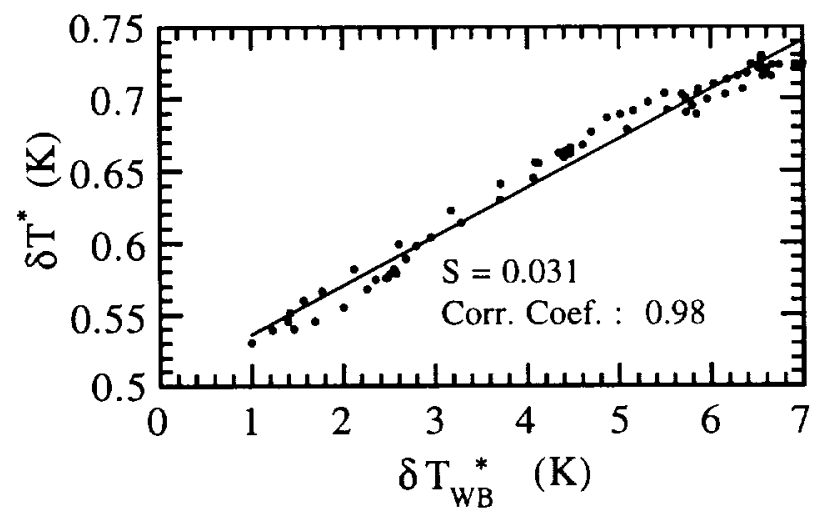

Figure 4: Regression relation between $\delta T^{*}$ and $\delta T^{*}{ }_{W B}$ for the three satellite overlaps NOAA $10-11$, NOAA $12-11$, and NOAA $12-14$ (see text for details). 


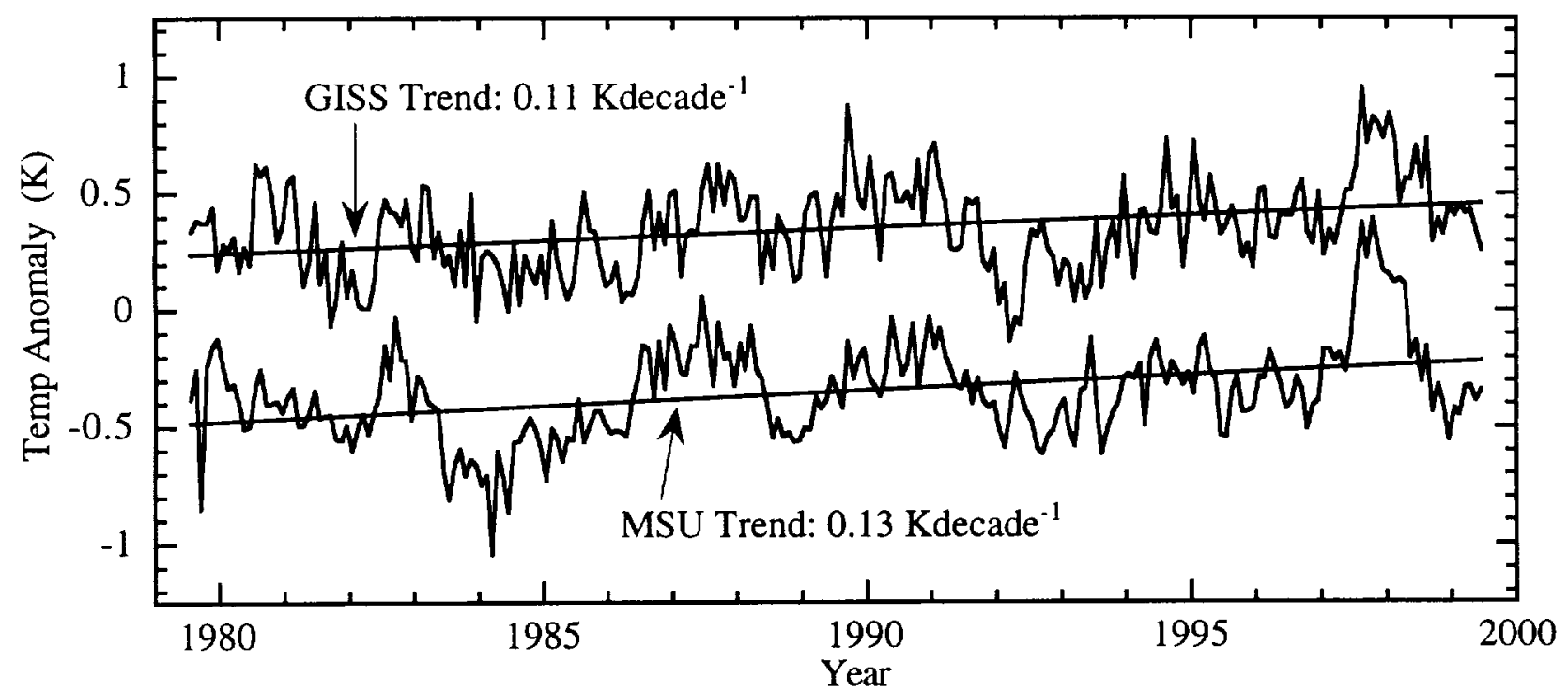

Figure 5: Anomaly time series and trend of MSU Ch 2 global monthly-mean temperature for the time period 1980 to 1999 is compared with corresponding information deduced from the conventional data analysis made by GISS. Note for clarity that the MSU and GISS time series are offset by $0.7 \mathrm{~K}$.

In Fig. 5, we show monthly-mean temperature anomalies of Time Series $\mathbf{A}$ after correction with $\mathrm{S}$ equal to 0.03 . From this time series, we find that the vertically-weighted global-mean temperature of the atmosphere, with a peak weight near the mid troposphere, increased by $0.13 \mathrm{Kdecade}^{-1}$ during the period 1980 to 1999.

We estimate the total error in the global temperature trend to be $0.05 \mathrm{Kdecade}^{-1}$. This error includes uncertainties in the overlap adjustment between NOAA 9 and 10 , and the value of S. It also includes errors introduced by variations of hydrometeors in the atmosphere and surface emissivity (see Prabhakara et al., 1995). With this error, the MSU estimate of the global temperature trend is $0.13 \pm 0.05 \mathrm{Kdecade}^{-1}$.

In Fig. 5, we also show the surface temperature anomaly time series deduced by Goddard Institute for Space Studies (GISS) from conventional data corrected for urbanization effects (see Hansen et al., 1999). This time-series has a trend of $0.11 \mathrm{Kdecade}^{-1}$. The two time series shown in Fig. 5 have similar interannual variability. From this analysis, we find the global warming estimated from conventional meteorological data that have been corrected for urbanization effects is in reasonable accord with the satellite-deduced result.

\section{References}

Christy, J.R., R.W. Spencer, and E.S. Lobl, 1998: Analysis of the merging procedure for the MSU daily time series. J. Climate, 11 . 2016-2041.

Christy, J.R., R.W. Spencer, and W.D. Braswell, 2000: Calculating global atmospheric temperature from the MSU, a never ending story? Preprint Vol. of AMS 10th Conf. on Sat. Meteorology and Oceanography, January 2000 Long Beach, Cal., 396-399.

Gaffen, D.J., M.A. Sargent, R.E. Habermann, and J.R. Lanzante, 2000: Sensitivity of tropospheric and stratospheric temperature trends to radiosonde data quality, J. Climate. 13, 1776-1796.

Hansen, J., R. Ruedy, M. Sato, and R. Reynolds, 1996: Global surface air temperature in 1995: Return to pre-Pinatubo levels. Geophys. Res. Lett., 23, 1665-1668, 1996.
Hansen J., R. Ruedy, J. Glascoe, and M. Sato, 1999: GISS analysis of surface temperature change. J. Geophys. Res, 104, 30997-31022. (see http:/www.giss.nasa.gov/data/update/gistemp/GLB.Ts.txt)

Hurrell, J.W. and K.E. Trenberth, 1997: Spurious trends in the satellite MSU temperature trends arising from merging different satellite records. Nature, 386, 164-167.

Hurrell, J.W. and K.E. Trenberth, 1998: Difficulties in obtaining reliable temperature trends: Reconciling the surface and satellite MSU 2R trends. J. Climate, 11, 945-967.

Hurrell, J.W., S.J. Brown, K.E. Trenberth, and J.R. Christy, 2000: Comparison of tropospheric temperatures from radiosondes and satellites: 1979-98. Bull. Amer. Met. Soc., 81, 2165-2177.

Jones, P.D., M. New, D.E. Parker, S. Martin, and I.G. Rigor, 1999: Surface air temperature and its change over the past 150 years. Rev. Geophys., 37, 173-199.

Mo, T., 1995: A study of the Microwave Sounding Unit on the NOAA12 satellite. IEEE Trans. Geosci.and Rem. Sens., 33, 1141-1152.

NOAA, 1998: NOAA Polar-Orbiter Data Users Guide. (Available from http://www2.ncdc.noaa.gov/docs/podug/)

Prabhakara, C., J.J. Nucciarone, and J.-M.Yoo 1995: Examination of 'Global atmospheric temperature monitoring with satellite microwave measurements': 1) Theoretical considerations. Climatic Change, 30 , 349-366.

Prabhakara, C., R. Iacovazzi, Jr., and J.-M. Yoo, 1998: Global warming deduced from MSU. Geophys. Res. Lett., 25, 1927-1930.

Prabhakara, C., and R. Iacovazzi, Jr., 1999: Comments on "Analysis of the merging procedure for the MSU daily temperature time series." J. Climate, 12, 3331-3334.

Spencer, R.W. and J.R. Christy, 1990: Precise monitoring of global temperature trends from satellites. Science, 247, 1558-1562.

Trenberth, K.E. and J.W. Hurrell, 1997: How accurate are satellite 'thermometers'. Nature, 389, 342-343.

Wentz, F.J. and M. Schabel, 1998: Effects of orbital decay on satellitederived lower-tropospheric temperature trends. Nature, 394, 661664.

C. Prabhakara, Code 913, NASA Goddard Space Flight Center, Greenbelt, MD, 20771. (e-mail: cuddapah@climate.gsfc.nasa.gov)

(Received April 25, 2000; revised September 18, 2000; accepted September 19, 2000) 